\title{
Recent ECRH Experiments in the L-2 M Stellarator with the Use of a New High-Power Gyrotron*)
}

\author{
Nikolay K. KHARCHEV, German M. BATANOV, Mikhail S. BEREZHETSKII, \\ Valentin D. BORZOSEKOV, Oleg I. FEDYANIN, Stanislav E. GREBENSHCHIKOV, \\ Iina A. GRISHINA, Yuriy V. KHOL'NOV, Leonid V. KOLIK, Eugeny M. KONCHEKOV, \\ Lev M. KOVRIZHNYKH, Natalia F. LARIONOVA, Dmitriy V. MALAKHOV, \\ Aleksey I. MESHCHERYAKOV, Alexander E. PETROV, Eugeniy I. PLESHKOV, \\ Karen A. SARKSYAN, Sergey V. SHCHEPETOV, Nina N. SKVORTSOVA, \\ Vladimir D. STEPAKHIN, Ildar Yu. VAFIN, Dmitriy G. VASILKOV and Genadiy S. VORONOV \\ General Physics Institute Russian Academy of Science, Moscow Russia
}

(Received 3 December 2010 / Accepted 21 February 2011)

\begin{abstract}
The results of experiments on plasma confinement at ECRH power density up to $1.5 \mathrm{MW} / \mathrm{m}^{3}$ are reported. It is shown that the results of measurements of the diamagnetism and poloidal rotation velocity of the plasma in the peripheral region agree with numerical calculations using a neoclassical model with allowance for anomalous losses. The correlation has been found between the low-frequency modulation of the gyrotron power radiation and the modulation of the radiation scattered by the turbulent plasma density fluctuations.
\end{abstract}

(c) 2011 The Japan Society of Plasma Science and Nuclear Fusion Research

Keywords: stellarator, gyrotron, microwave, power density, locally trapped particle, anomalous loss

DOI: $10.1585 /$ pfr.6.2402142

\section{Introduction}

In the problem of transport in high-temperature plasma, some issues related to anomalous (turbulent) transport and the issues related to transport by locally trapped particles at low collision frequencies are still the focus of attention in stellarator physics. The resolution of these issues requires performing experiments at large values of heating power densities.

The construction of a new gyrotron complex is well under way for ECRH experiments in the L-2 M stellarator. The complex consists of two GYCOM/IAP three-electrode gyrotrons with recovery of the energy of an electron beam and is intended for ECR heating of plasma at the second harmonic of the electron gyrofrequency.

The first gyrotron has a power up to $800 \mathrm{~kW}$ and efficiency of energy conversion of the electron beam into high-energy microwave radiation of $75 \%$ (frequency of $75 \mathrm{GHz}$ ). The second gyrotron has a power of $700 \mathrm{~kW}$ and efficiency of $60 \%$ can operate at three fixed frequencies: $71.5,74.8$, and $78.2 \mathrm{GHz}$. Therefore, total ECRH power density in L-2 $\mathrm{M}$ can reach a level above $5 \mathrm{MW} / \mathrm{m}^{3}$. At present, the first stage of the complex with a single gyrotron is completed. The gyrotron is put into operation with a power of $500 \mathrm{~kW}$ (with input power density of $1.9 \mathrm{MW} / \mathrm{m}^{3}$ ). For an average plasma density of $2 \cdot 10^{19} \mathrm{~m}^{-3}$, this value corresponds to a rate of heating of

author'se-mail: khar@fpl.gpi.ru

*) This article is based on the presentation at the 20th International Toki Conference (ITC20). plasma electrons as high as $600 \mathrm{keV} / \mathrm{s}$ per electron.

Below is a summary report on experiments with the use of the new gyrotron complex. The energy balance was performed on the basis of measurements plasma diamagnetism and Pfirsch-Schlueter currents. The ECRH power was varied from 100 to $500 \mathrm{~kW}$, and the plasma density was varied from $1 \cdot 10^{19} \mathrm{~m}^{-3}$ to $3 \cdot 10^{19} \mathrm{~m}^{-3}$.

\section{Experimental Results}

Figure 1 compares two series of typical signals measured at two values of absorbed microwave power of $130 \mathrm{~kW}$ and $360 \mathrm{~kW}$ and the same average plasma density of $\sim 1.7 \cdot 10^{19} \mathrm{~m}^{-3}$. Gas breakdown occurs immediately after the leading edge of the gyrotron pulse. The average plasma density over the central chord attains a maximal value in $1.5-2 \mathrm{~ms}$ and then decreases slowly by $20-30 \%$ by the midpoint of the microwave pulse. Then, the density smoothly increases by $10-15 \%$ toward the end of the heating pulse and continues to increase after ECRH pulse ends. Such a difference in the time behavior of the average plasma density early in the heating pulse and after its end is due to redistribution of the plasma density in the plasma column, i.e., flattening of the radial profile after breakdown and narrowing after the end of the microwave pulse. The density increase in the latter part of the ECRH pulse may be attributed to increased gas flow from the chamber walls. As is seen in Fig. 1, a doubling of the heating power does not affect the time evolution of the density in a qualitative sense. 

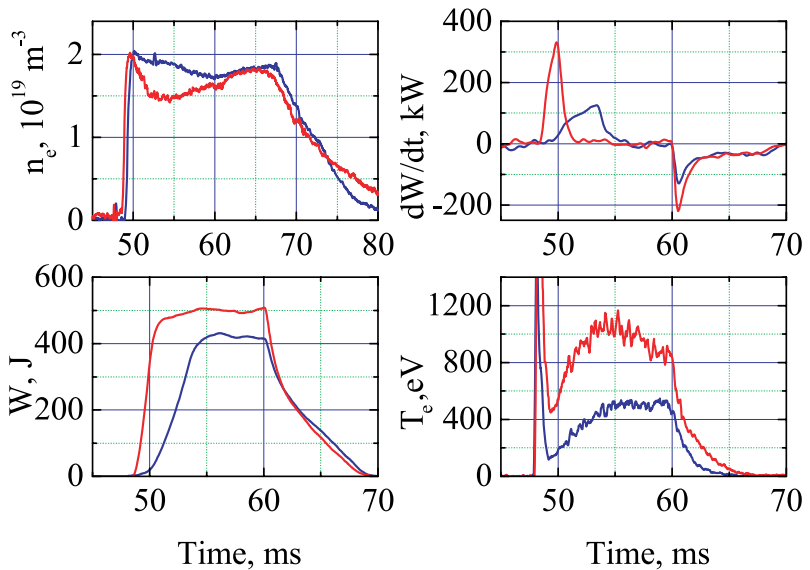

Fig. 1 Typical time behavior of the plasma signals in two shots: a) average density, b) derivative of a diamagnetic signal, c) plasma energy, d) central electron temperature. Blue curves are for shot 64333 , where the absorbed power is $130 \mathrm{~kW}$. Red curves are for shot 64194 , where the absorbed power is $360 \mathrm{~kW}$.

The plasma energy, measured using diamagnetic loops, increases rapidly after gas breakdown; then it increases but at a slower rate and approaches a steady state by the midpoint of the microwave pulse. Some increase in the latter part of the heating pulse is due to insignificant increase in the density at that time. A doubling of the heating power leads to a sharp reduction in the time during which the energy reaches its quasi-steady state. After the heating pulse is switched off, the energy falls, while the plasma density varies slightly. At the same time, power losses (the derivative of the diamagnetic signal) drops in 2-3 ms to the level of radiative power losses.

The central electron temperature, measured from the intensity of electron cyclotron emission at the second harmonic of the electron gyrofrequency $(76 \mathrm{GHz})$ usually increases during the early part of the ECRH pulse and then decreases slowly toward the end of the microwave pulse, if the plasma density increases.

Figure 2 shows the measured values of plasma energy for the midpoint of ECRH pulse (54-55 ms) for two values of plasma density $\left(1.6-1.8 \cdot 10^{19} \mathrm{~m}^{-3}\right.$ and $2.0-$ $2.2 \cdot 10^{19} \mathrm{~m}^{-3}$ ). Also shown are the dependences of the plasma energy on the absorbed power, which were calculated with the use of the model $[1,2]$ for the same density values. It is impossible to give detailed description of this model for reason in space, and we may only refer the reader to the works $[1,2]$. Here, we only note that, for the neoclassical transport coefficients for the stationary case, we have derived a system of four nonlinear firstorder equations for the density, temperature, and ambipolar field. Anomalous losses were accounted for by a purely phenomenological model on the basis of dimensional considerations and comparison with experimental data. The results of calculation of the energy confinement time with this model in a wide range of parameters correspond to

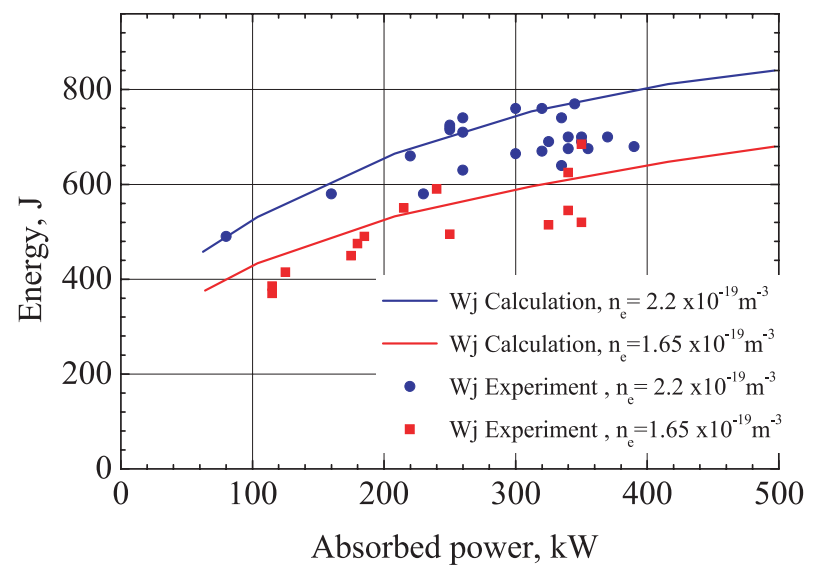

Fig. 2 Calculated (lines) and experimental (points) plasma energy values for two plasma densities: $2.2 \times 10^{-19} \mathrm{~m}^{-3}$ (blue) and $1.65 \times 10^{-19} \mathrm{~m}^{-3}$ (red).

the International Stellarator Scaling ISS04 [3]. It is seen from data in Fig. 2, the spread in experimental values of the plasma energy about the calculated curves is no more than $20-25 \%$. We regard it as a satisfactory agreement between calculations and experiment. It should be noted that, in the range of ECRH powers and plasma densities used in our experiments, trapped electrons might play a significant role in heat conduction processes. And because of the outward Shafranov shift of the magnetic axis by more than $3 \mathrm{~cm}$, the role of trapped electrons might increase in importance in comparison with the model used in calculations. This circumstance, for example, might explain the fact the electron temperature values measured in the experiment are lower than those predicted by the model.

The radial profile of the ambipolar electric field is of considerable importance in determining the mechanisms of heat transport. With the aim of determining the values of the radial field by means of optical spectroscopy we have measured the poloidal velocity of plasma rotation from Doppler shifts of the boron and carbon spectra in the edge plasma. Figure 3 shows the electric field profiles calculated from the measured rotation velocity for two values of heating power of 200 and $400 \mathrm{~kW}$ in the neglect of the density and temperature gradients. This approximation is fully justified for the pedestal region $(r / a<0.95)$ in the $\mathrm{H}-$ mode with an edge transport barrier, when the density and temperature gradients are located in the barrier region.

The experimental results show that the electric field is negative in the edge plasma. The field strength is about $-5 \mathrm{kV} / \mathrm{m}$ and changes to $-10 \mathrm{kV} / \mathrm{m}$ with increasing heating power. The field strength decreases towards the center. As the power increases, a point where the field vanishes $(E=$ $0)$ shifts toward the center.

In Fig. 3, also shown are the results of numerical computations of the electric field with the TRANSZ code [4]. As can be seen from the figure, the both sign and magnitude of the field strength are close to the results of the experiment. However, the point where $E=0$ in the model 


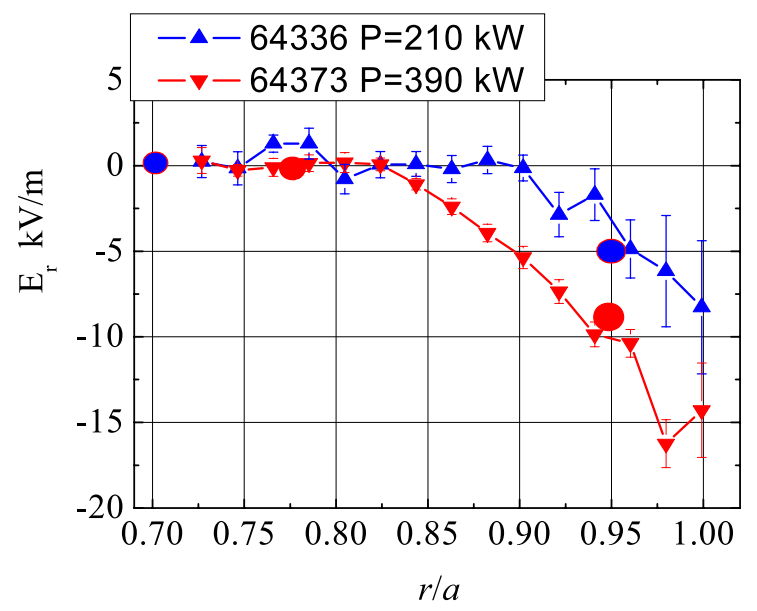

Fig. 3 Electric field at the plasma edge for ECRH powers of 210 and $390 \mathrm{~kW}$. Filled circles show the results of numerical computations.

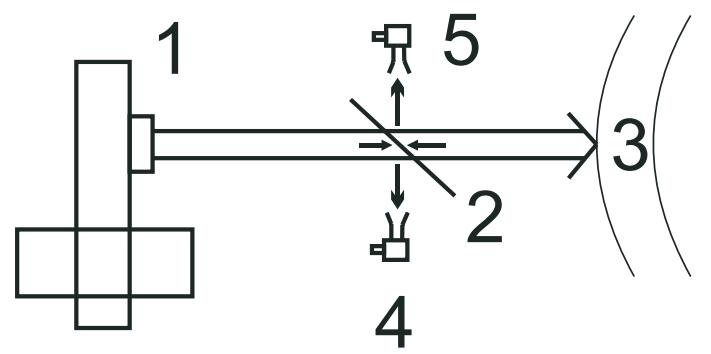

Fig. 4 Quasioptical coupler: 1 gyrotron, 2 semitransparent mirror (mica plate), 3 plasma, 4 detector of incident radiation, 5 detector of scattered radiation.

is shifted outward from the center.

Previously in [5], we revealed a correlation between low-frequency modulation of the gyrotron power and turbulent fluctuations of the plasma density. This phenomenon may have an impact upon efficiency of EC heating because of an onset of microturbulence as a result of positive feedback in the gyrotron-plasma system.

When measuring envelops of gyrotron radiation power and reflected radiation from the plasma, we used the scheme with a quasioptical coupler (Fig. 4). Figure 5 illustrates signals of detectors receiving a direct signal (inherent radiation) and signal of reflected radiation. Backward reflected signal corresponds to backward $\left(180^{\circ}\right)$ scattering radiation of the gyrotron, i.e., according to the Bragg condition, the wavenumber of fluctuations involved in scattering is $k_{\mathrm{s}}=2 K_{0}=31.4 \mathrm{~cm}^{-1}$ (where $K_{0}$ is the wavenumber of incident radiation).

Figure 6 demonstrates the time evolution of the crosscorrelation function of these signals. Integration was over a 2-ms time window. The cross-correlation coefficient exceeds $50 \%$. The large value of this coefficient indicates that the gyrotron output power is affected by the microwave radiation coming from the plasma. Displacement of one sig-

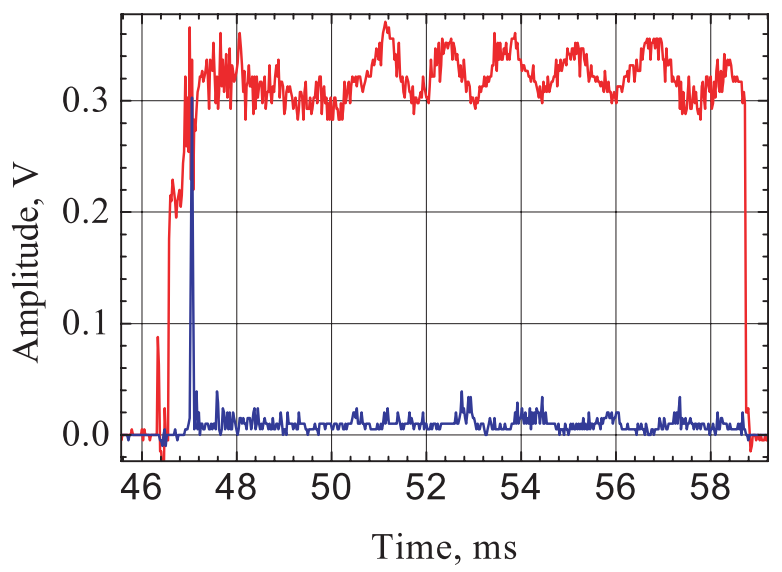

Fig. 5 Gyrotron power signal (red) and scattered-radiation signal (blue).

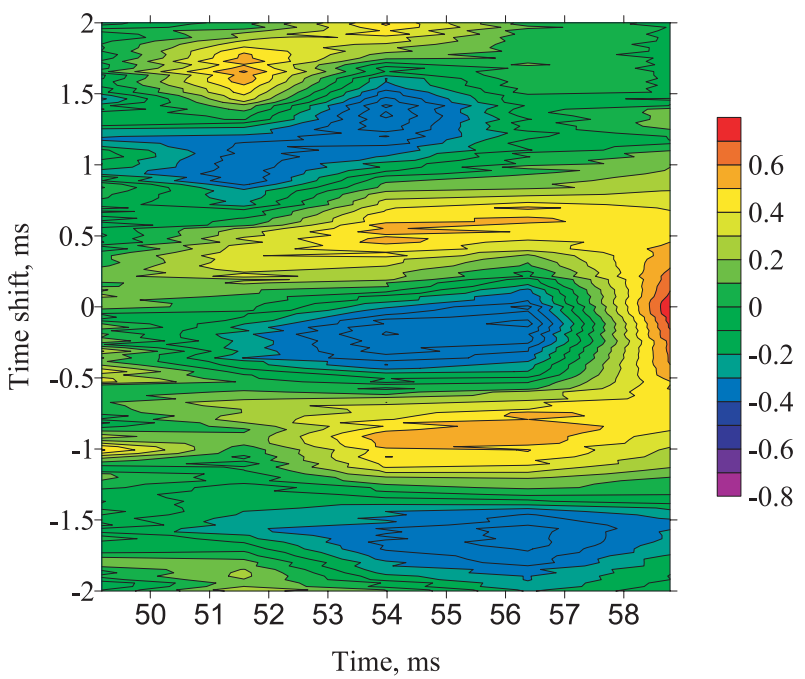

Fig. 6 Cross-correlation coefficient between envelops of the gyrotron power and the backward scattered radiation due plasma density fluctuations.

nal with respect to another on time axis discloses that the reflected radiation is in antiphase with the incident radiation.

\section{Conclusions}

To summarize results obtained at the L-2 M stellarator in measurements of EC heating of currentless plasma at the second harmonic of the gyrotron frequency $(75 \mathrm{GHz})$ in the range of heating-power densities from $0.38 \mathrm{MW} / \mathrm{m}^{3}$ to $1.5 \mathrm{MW} / \mathrm{m}^{3}$ :

(1) We have established that, within limits of statistical straggling of experimental data on plasma diamagnetism, the observed increase in the plasma energy with ECRH power agrees with numerical calculations of neoclassical losses taking into account an important role of locally trapped particles and anomalous losses in heat transport; 
(2) We have carried out measurements of the poloidal velocities of plasma rotation from Doppler shift of spectral lines of boron and carbon ions at the periphery of the plasma column. These data were used to estimate the ambipolar electric field. It is shown that the both direction and strength of the field observed experimentally are in agreement with calculations using the hybrid model (neoclassical losses plus anomalous losses);

(3) For a three-electrode gyrotron with a diode gun, there exists a correlation between the gyrotron power mod- ulation and the power modulation in the signal of backward scattered radiation owing to plasma fluctuations.

[1] L.M. Kovrizhnykh, Plasma Phys. Rep. 32, 988 (2006).

[2] L.M. Kovrizhnykh, Plasma Phys. Rep. 34, 529 (2008).

[3] H. Yamada et al., Nucl. Fusion 45, 1684 (2005).

[4] S.E. Grebenshchikov, I.S. Danilkin and A.B. Mineev, Plasma Phys. Rep. 22, 551 (1996).

[5] G.M. Batanov et al., Tech. Phys. 45, 566 (2001). 